\title{
COMMENT \\ Different flavors of IL-21 in regulation of intestinal IgA to
} commensals

\author{
Yingzi Cong ${ }^{1,2}$ and Yanqing $\mathrm{Li}^{3}$ \\ Mucosal Immunology (2019) 12:36-38; https://doi.org/10.1038/s41385-018-0099-z
}

IL-21 has been shown to regulate IgA production. However, how it regulates gut microbiota and intestinal homeostasis through regulation of $\lg \mathrm{A}$ is still not fully understood. A study by Cho et al. in this issue of Mucosal Immunology ${ }^{1}$ demonstrates that IL-21 promotes intestinal $\operatorname{lgA}$ responses to atypical commensals to reshape the mucosal immunity and microbiota.

The intestines harbor a microbiota consisting of 100 trillion microbes in humans. Over millions of years of evolution, hosts have developed multiple regulatory mechanisms to keep the vast microbial community in check. Gut microbiota, in turn, has a major impact on host systems, particularly on the development of the intestines and the immune system. Despite the enormous bacterial challenge, the intestines live in harmony with the microbiota, in part due to interactions of the microbiota with the host to maintain a symbiotic relationship. The host senses and responds to the microbiota through coordinated immune responses that are not overly aggressive towards commensal microbes, but remain primed to defend against invading pathogens. Among the multiple regulatory mechanisms that have been evolved by the host, Th17 cells and intestinal $\lg A(\lg A)$, which are enriched in intestines, have crucial roles in the maintenance of intestinal homeostasis against the microbiota. ${ }^{2,3}$

As the most abundant antibody isotype in the intestine, IgA not only functions to neutralize and aid in clearance of toxins and extracellular pathogens through high affinity binding, ${ }^{4}$ but also binds to commensal bacteria through the Fab as well as through glycosyl interactions to limiting contact of gut microbiota and its antigens with the hosts. ${ }^{5} \operatorname{lgA}$ regulates the microbiota, and gut bacteria in turn adapt to $\lg \mathrm{A}$ by altering their gene expression patterns to promote survival and homeostasis within the intestines. $^{5}$ The high level of IgA production in the intestines is driven by microbial colonization, which is exemplified by the fact that germ-free mice have low levels of $\lg A$ and $\lg A$-producing $B$ cells with subsequent recolonization by microbiota restoring $\lg A$ production. ${ }^{6}$ Multiple mechanisms regulate intestinal $\lg A$ responses, including both $T$ cell-dependent and $T$ cellindependent pathways. ${ }^{7}$ Although the relative contribution of $\mathrm{T}$ cell-dependent and T cell-independent regulation of intestinal $\lg \mathrm{A}$ responses is still not completely understood, a recent report demonstrated that bacteria in the small intestine but not indigenous colonic bacteria dictated induction of specific $\lg \mathrm{A}$ in a $T$ cell-independent manner, although the mechanisms remain unclear. $^{8}$ Most commensals elicited strong T-independent responses that originated from the $\mathrm{B} 1 \mathrm{~b}$ and $\mathrm{B} 2$ cells, but excluded natural antibacterial B1a specificities. However, atypical commensals, such as segmented filamentous bacteria (SFB), Mucispirillum, and Helicobacter, evaded $\mathrm{T}$ cell-independent but elicited $\mathrm{T}$ celldependent IgA responses. However, how the differential $\lg \mathrm{A}$ responses to atypical commensals contribute to intestinal homeostasis are still not fully clear.

The intestinal tract has been shown to be a natural site for the development of Th17 cells, which produce a distinct set of cytokines, including IL-17, IL-21, and IL-22. Intestinal Th17 cell development is stimulated by specific species of microbiota, with SFB being identified as one of such stimulators. Interestingly, SFB also stimulates intestinal $\lg A$ production. ${ }^{9}$ Although both pro- and anti-inflammatory functions of Th17 cells have been demonstrated in different experimental systems, the enrichment of Th17 cells in the intestines suggests a role for these cells in mucosal homeostasis and more specifically in the containment of the vast local microbiota. Th17 cells induce mucosal IgA production in the intestines directly or through conversion to T follicular helper (Tfh) cells via production of IL-17 and IL-21. ${ }^{10,11}$ Intestinal IgA is

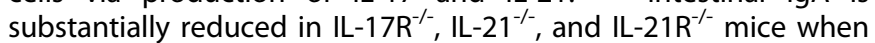
compared to wild-type mice, and IL-21 induces IgA class-switch recombination in cooperation with TGF $\beta$ and retinoic acid in vitro. ${ }^{10}$ However, as IL-21 is predominantly produced by Th17 and Tfh cells, it remains unclear which source of IL-21 is most potent in inducing intestinal IgA production. It is also unclear how IL-21 regulates IgA responses to atypical commensal bacteria. Furthermore, both pro-inflammatory and anti-inflammatory functions for IL-21 in the intestines have been reported, thus, how it affects intestinal homeostasis remains unclear. In this issue of Mucosal Immunology, ${ }^{1}$ Cho et al. found that IL-21 was critical for an $\lg A$ response against atypical commensals but not to the majority of commensals. Additionally, a defective IgA response to atypical commensals in the absence of IL-21 signaling led to higher bacterial burdens and subsequent expansion of Th17 cells. Strikingly, the microbiota-mediated secondary changes enhanced $T$ cell responses in the mucosa and dampened enteric infectioninduced immunopathology in the intestines.

Although both Th17 and Tfh cells produce high levels of IL-21, how these T cells produce IL-21 in response to microbiota in the intestines is still not well established. Cho et al. found that CD4 ${ }^{+}$ $\mathrm{T}$ cells are the main source of IL-21 production in the intestine, mainly in the Peyer's patches (PPs) and small intestine lamina propria (SILP). Atypical commensal SFB colonization stimulated production of IL-21, mainly in CD4 T cells. Surprisingly, although

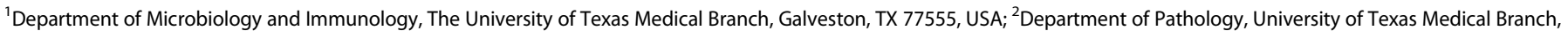
Galveston, TX 77555, USA and ${ }^{3}$ Department of Gastroenterology, Qilu Hospital, Shandong University, Jinan, Shandong, China

Correspondence: Yingzi Cong (yicong@utmb.edu) or Yanqing Li (liyanqing@sdu.edu.cn)

Received: 13 August 2018 Revised: 19 September 2018 Accepted: 28 September 2018

Published online: 25 October 2018 


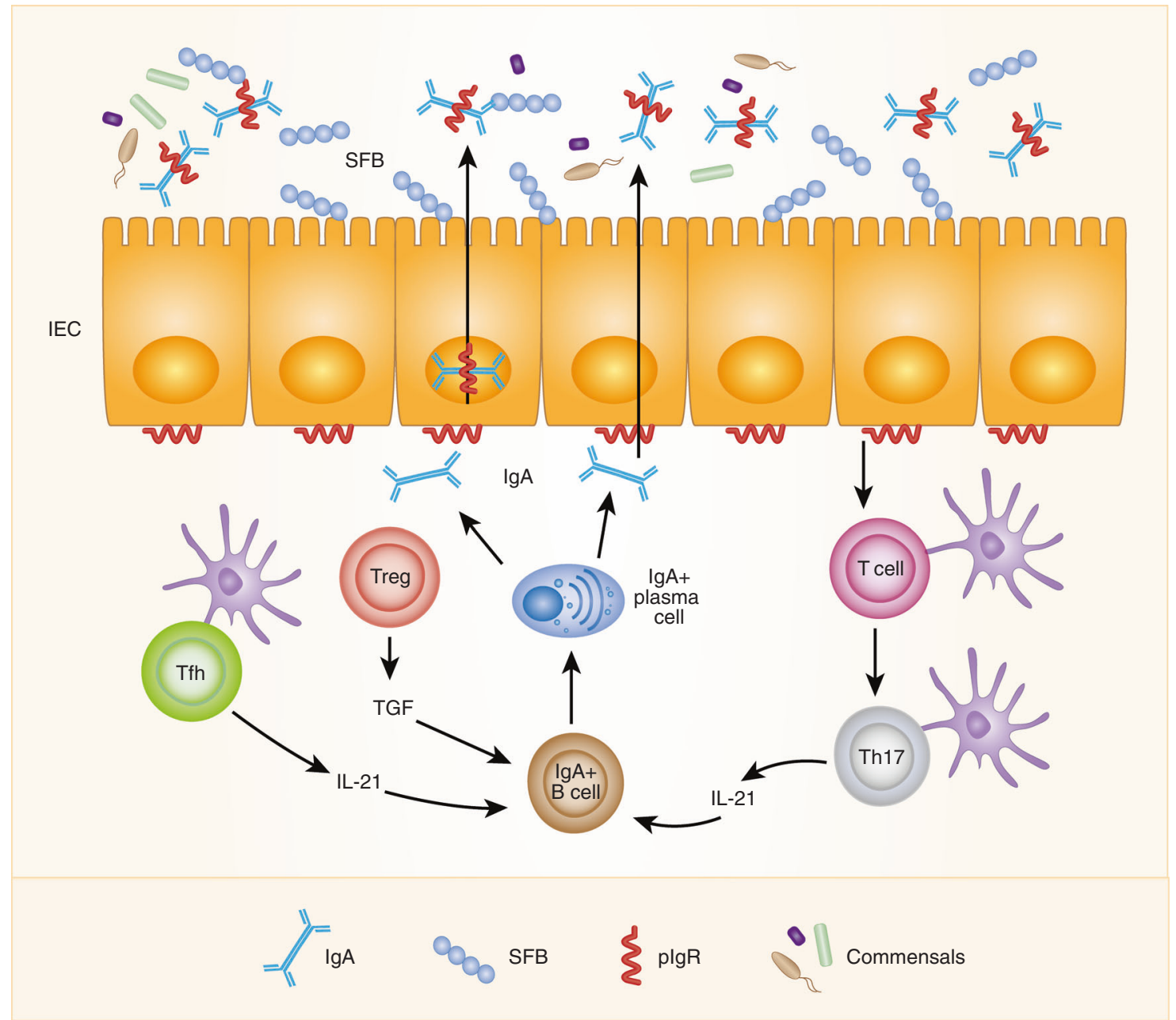

Fig. 1 IL-21 promotes IgA responses in the intestine. SFB adhesion to intestinal epithelial cells (IEC) promotes T cells to differentiate into Th17 cells which produce IL-17 and IL-21. Tfh cells also produce IL-21. IL-21, in cooperation with TGF $\beta$ produced by DC and Tregs, induces IgA classswitch recombination to promote B cell IgA production. Once translocated through IEC into intestinal lumen by binding polymeric lg receptor (plgR), IgA constrains SFB, and other atypical commensal colonization, which in turn regulates mucosal immunity to luminal antigens and enteric pathogen infection

some Tfh cells expressed IL-21 in the absence of SFB, the frequency of Tfh cells was reduced following colonization with SFB, indicating that the majority of IL-21-producing cells in the intestines are not Tfh cells, but likely Th17 cells. As both Th17 and Tfh cells promote intestinal IgA responses in the intestines, this important finding will clarify the relative contributions of these cells in regulation of intestinal IgA response to commensals. When Cho et al. examined $\lg \mathrm{A}^{+} \mathrm{B}$ cells in PPs of both $\mathrm{SFB}^{+}$and $\mathrm{SFB}^{-}$ IL21R KO mice and their littermate controls, they found that $\lg \mathrm{A}^{+}$ $B$ in PPs of SFB ${ }^{+}$IL-21R KO mice were significantly decreased compared to WT mice. IL-21 signaling plays a critical role in intestinal IgA class switch recombination (CSR) in vivo, in that the number of germinal center B cells in the PPs of IL-21R KO mice was dramatically decreased compared to WT mice, whereas the number of Th cells remained unchanged. This is despite the fact that IL-21 has been shown to promote Tfh cells in peripheral lymph tissues. However, there is no difference of the numbers of $\operatorname{lgA}^{+} B$ cells both in the PPs and SILP in SFB ${ }^{-}$IL-21R KO mice compared to WT mice. Thus, Cho et al. identified an important role of IL-21 in promoting IgA response to atypical but not the majority of commensal microbiota. As a consequence, defective $\operatorname{lgA}$ response in $\mathrm{SFB}^{+}$IL-21R KO mice resulted in significantly higher levels of SFB, Helicobacter and Mucispirillum compared to SFB ${ }^{+}$WT mice, confirming the crucial role of intestinal $\lg A$ in constraining bacterial colonization in the intestines.

IL-21 has been well-established for its role in amplifying and maintaining Th17 cells while inhibiting Treg cell differentiation. ${ }^{12}$ However, whether this machinery of Th17 cell development is present in the intestine, especially in response to atypical commensals, is still not clear. Cho et al. found that the frequency of both Th17 cells and Tregs was increased in SILP but not LILP of IL-21R KO mice compared to WT mice, consistent with SFB colonization mainly in ileum. However, there was no difference in Th17 and Tregs between SFB ${ }^{-}$IL-21R KO mice and WT mice. SFB is well known for its ability to induce Th17 cells in the intestines, and higher levels of SFB in SFB ${ }^{+}$IL-21R KO mice could lead to the expansion of Th17 cells, however, whether the increased Tregs is due to lack of IL-21 signaling thus releasing IL-21 inhibition on Tregs or due to stimulation of the altered microbiota was still unclear. What is the consequence of defective IgA-regulated microbiota changes in IL-21R KO mice with atypical commensals? Cho et al. found those mice developed an increased mucosal Th17 response to oral antigen OVA. Even more strikingly, $\mathrm{SFB}^{+}$ Helicobacter $^{+}$IL-21R KO mice but not SFB ${ }^{-}$Helicobacter IL-21R $^{-212}$ $\mathrm{KO}$ mice demonstrated reduced intestinal pathology upon infection with Citrobacter rodentium, indicating that IL-21-lgA- 
regulated microbiota changes reshape the intestinal responses to mucosal antigen stimulation and infection.

The study by Cho et al. thus, provides great insights into the role of IL-21 through regulation of IgA in intestinal homeostasis, mucosal immunity, microbiota, and defense against enteric pathogens (Fig. 1). As with all elegant studies, it also raises many questions. It is unclear why IL-21 signaling only regulates intestinal $\lg A$ response to atypical commensals but not the majority of commensals. Also, why does a defective IgA response in IL-21R KO mice colonized with atypical commensals only lose the ability to constrain atypical commensals but not other commensals? What is the benefit for hosts to develop such mechanisms in maintaining the intestinal homeostasis? It has been shown that IL-21 serves as an amplifying cytokine for Th17 cell development, ${ }^{12}$ thus it remains unclear how increased SFB drives the expansion of Th17 cells in the absence of IL-21 signaling in $\mathrm{SFB}^{+}$mice. In the absence of $\mathrm{IL}-21$, are there compensational mechanisms for amplifying Th17 cell differentiation in context with the atypical commensals in the intestines? Do atypical commensals and enteric pathogens interact to regulate intestinal inflammation? Investigations into these questions will provide great insight into how IL-21 regulates host responses to microbiota and infection, thus providing a new avenue for therapeutic treatment of inflammatory bowel disease and other sources of intestinal inflammation.

\section{ACKNOWLEDGEMENTS}

This work was supported by NIH grants DK105585 and DK112436.

\section{ADDITIONAL INFORMATION}

Competing interests: The authors declare no competing interests.

\section{REFERENCES}

1. Cho, H. et al. Defective IgA response to atypical intestinal commensals in IL-21 receptor deficiency reshapes immune cell homeostasis and mucosal immunity. Mucosal Immunol., https://doi.org/10.1038/s41385-018-0056-x (2018).

2. Colliou, N. et al. Commensal propionibacterium strain UF1 mitigates intestinal inflammation via Th17 cell regulation. J. Clin. Invest. 127, 3970-3986 (2017).

3. Slack, E., Balmer, M. L. \& Macpherson, A. J. B cells as a critical node in the microbiota-host immune system network. Immunol. Rev. 260, 50-66 (2014).

4. Corthesy, B. Multi-faceted functions of secretory $\lg$ A at mucosal surfaces. Front. Immunol. 4, 185 (2013).

5. Nakajima, A. et al. IgA regulates the composition and metabolic function of gut microbiota by promoting symbiosis between bacteria. J. Exp. Med. 215, 2019-2034 (2018)

6. Benveniste, J., Lespinats, G. \& Salomon, J. Serum and secretory IgA in axenic and holoxenic mice. J. Immunol. 107, 1656-1662 (1971).

7. Lycke, N. Y. \& Bemark, M. The regulation of gut mucosal IgA B-cell responses: recent developments. Mucosal Immunol. 10, 1361-1374 (2017).

8. Bunker, J. J. et al. Innate and adaptive humoral responses coat distinct commensal bacteria with Immunoglobulin A. Immunity 43, 541-553 (2015).

9. Lecuyer, E. et al. Segmented filamentous bacterium uses secondary and tertiary lymphoid tissues to induce gut $\lg A$ and specific $T$ helper 17 cell responses. Immunity 40, 608-620 (2014).

10. Cao, A. T. et al. Interleukin (IL)-21 promotes intestinal IgA response to microbiota. Mucosal Immunol. 8, 1072-1082 (2015).

11. Hirota, K. et al. Plasticity of Th17 cells in Peyer's patches is responsible for the induction of T cell-dependent IgA responses. Nat. Immunol. 14, 372-379 (2013).

12. Korn, T. et al. IL-21 initiates an alternative pathway to induce proinflammatory $T$ (H)17 cells. Nature 448, 484-487 (2007). 